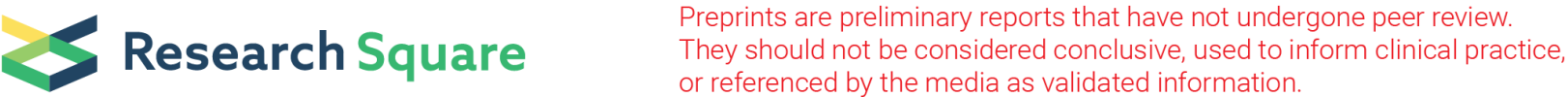

\section{Molecular allergen sensitization of Aspergillus fumigatus in allergic bronchopulmonary aspergillosis in Guangzhou, Southern China}

Wenting Luo

Guangzhou Medical University

Haisheng Hu ( $\Delta$ 781640613@qq.com )

Guangzhou Medical University https://orcid.org/0000-0001-7873-6956

Zehong Wu

Guangzhou Medical University

Nili Wei

Guangzhou Medical University

Huimin Huang

Guangzhou Medical University

Peiyan Zheng

Guangzhou Medical University

Baoqing Sun

Guangzhou Medical University

Yong Liu

Guangzhou Medical University

\section{Research article}

Keywords: Molecular allergen, Aspergillus fumigatus, Allergic bronchopulmonary aspergillosis, Aspergillus fumigatus-sensitized asthma, Mycotic allergens

Posted Date: March 10th, 2020

DOl: https://doi.org/10.21203/rs.3.rs-16458/v1

License: (c) (i) This work is licensed under a Creative Commons Attribution 4.0 International License. Read Full License 


\section{Abstract}

Background Few studies have assessed the sensitization of mycotic allergens and Aspergillus fumigatus molecular allergen. This study aimed to investigate the connection between A.fumigatus components and mycotic allergens between allergic bronchopulmonary aspergillosis (ABPA) patients and A. fumigatus (Af)-sensitized asthma.

Methods Serum Penicillium chrysogenum, Cladosporium herbarum, Mucor racemosus , Candida albicans , Alternaria alternata , Helminthosporium halodes and A.fumigatus allergen components (Asp $f$ 1, Asp f 2, Asp f 3, Asp f 4, and Asp f 6) slgE level were measured via ImmunoCAP assay in 18 ABPA patients and 54 Af-sensitized asthma patients in Guangzhou city.

Results $94.44 \%$ of ABPA patients and $87.04 \%$ of Af-sensitized asthma patients were co-sensitization to at least one another fungal allergen. The positivity rates of $C$. albicans $(P<0.05)$ and A.alternata $(P<0.05)$ were higher in ABPA than that in Af-sensitized asthma patients. The positive rates of Asp $f 1$ (88.89\% vs $59.26 \%, P<0.05)$, Asp f 2 (66.67\% vs $33.33 \%, P<0.05)$, Asp f 4 (61.11\% vs $33.33 \%, P<0.05)$, and Asp $f 6$ $(66.67 \%$ vs $14.81 \%, P<0.001)$ in ABPA were higher than those in Af-sensitized asthma patients. Patients with ABPA had higher IgE levels of Asp $f 1(P<0.05)$, Asp $f 4(P<0.05)$ and Asp $f 6(P<0.001)$ than those of Af-sensitized asthma patients. A.fumigatus was strongly correlated with C.herbarum ( $\mathrm{r} s=$ 0.688 ) in ABPA and A.alternata ( $r s=0.692)$ in Af-sensitized asthma patients. Optimal scale analysis was show that ABPA was more relevant to Af-components. (Cronbach`s alpha $=90.7 \%$ )

Conclusion The A.fumigatus components and it's connection with various mycotic allergens were different in ABPA and Af-sensitized asthma patients. This findings can expected to help local doctors in the diagnosis and immunotherapy of fungal allergies.

\section{1.introduction}

Fungi are the most common microorganisms in the human living environment, and it is not only easy to cause respiratory tract infection and induced inflammatory response, but also cause severe allergic reaction. Studies have shown that Aspergillus, Alternaria, Candida, Cladosporium and Epicoccum are considered major sources of allergens worldwide. ${ }^{1,2}$ During reproduction, fungi release large amounts of spores and hyphal fragments in the air, which may cause immunoglobulin $E$ (IgE)-mediated respiratory allergic diseases, ${ }^{3}$ in particular Aspergillus fumigatus (Af)-sensitized asthma and allergic bronchopulmonary aspergillosis (ABPA). ${ }^{4}$

ABPA is a pulmonary disease caused by A.fumigatus, which pathogenesis is owing to allergic response against A.fumigatus colonising the airways rather than saprophytic or invasive of the fungi ${ }^{5-6}$. When patients with ABPA are exposed to fungi in the environment, they will show repeated wheezing, dyspnea, and even life-threatening in severe cases ${ }^{7}$. Due to the lack of effective clinical treatment, patients have heavy economic burden and poor quality of life. A European study shows that Af-sensitized asthma easy 
to develop into $\mathrm{ABPA}^{8-9}$, which may induced by molecular allergen of A.fumigatus. There are five major molecular allergens of A.fumigatus (Asp f 1, Asp f 2, Asp f 3, Asp f 4 and Asp $f$ 6, respectively). Among them, Asp $f 1$ is the most important protein of A.fumigatus. It secretes a lot after spore germination and early fungal invasived, which is related to fungal colonization and saprophytic. Asp $\mathrm{f} 2$ is a fibronectin, Asp $f 3$ is an epitope of peroxidase membrane protein, Asp $f 4$ is a glycosylated hydrolase and Asp $f 6$ is a manganese superoxide dismutase ${ }^{10-11}$. Our previous research shows that more than $18 \%$ asthmatic patients are sensitized to Asp $f 3^{12}$.

Although several studies have focused on the A.fumigatus sensitization in Southern China, ${ }^{13,14}$ the studies of the connection between various fungal allergens and A.fumigatus components was still lack, especially in Guangzhou, a beautiful and unique cultural city in southern China. In addition, various fungal allergens and A.fumigatus components maybe co-pathogenic and play an important role in ABPA or Af-sensitized asthma. Accordingly, in this study, we compared various fungal allergens and A.fumigatus major components between ABPA and Af-sensitized asthma patient, and expected to provide meaningful evidence for accurate diagnosis and for guiding disease treatment.

\section{2.materials And Methods 2.1 Study design}

This study included 18 ABPA patients and 54 Af-sensitized asthma patients from 4,033 general population (9-79 year old) who had undergone A.fumigatus allergen slgE tests during January 2016 to December 2017 in the Allergy Information Repository of the National Clinical Research Center for Respiratory Disease (AIR-NCRCRD, Guangzhou, Southern China).

The diagnosis of asthma was based on the Global Initiative for Asthma guidelines ${ }^{15}$, and the diagnostic criteria of ABPA was based on Agarwal et al. ${ }^{16}$ by respiratory specialist, the patients had undergone allergen-specific immunotherapy, parasitic infections, cancer and immunodeficiency was excluded. All patients provided written informed consent, and the study was approved by the ethics committee of the First Affiliated Hospital of Guangzhou Medical University (GYYY-2016-73).

\subsection{Serum allergen-specific IgE detection}

In 18 patients with ABPA and 54 patients with Af-sensitized asthma, serum slgE level of Penicillium chrysogenum, Cladosporium herbarum, Aspergillus fumigatus, Mucor racemosus, Candida albicans, Alternaria alternata, Helminthosporium halodes, total IgE and Aspergillus fumigatus components including Asp f 1, Asp f 2, Asp f 3, Asp f 4, and Asp f 6 slgE were tested by PhadiaCAP 1000 (Thermo Fisher Scientific, Sweden). SIgE concentrations of $0.35 \mathrm{kUA} / \mathrm{L}$ or more were defined as positive or sensitization to the allergen. According to the slgE levels, the reactivity was categorized quantitatively into six classes: Class 1 ( $\geq 0.35 \mathrm{kUA} / \mathrm{L}$ to $<0.70 \mathrm{kUA} / \mathrm{L}$ ), Class 2 ( $\geq 0.70 \mathrm{kUA} / \mathrm{L}$ to $<3.50 \mathrm{kUA} / \mathrm{L})$, Class 3 ( $\geq$ 
$3.50 \mathrm{kUA} / \mathrm{L}$ to $<17.50 \mathrm{kUA} / \mathrm{L})$, Class 4 ( $\geq 17.50 \mathrm{kUA} / \mathrm{L}$ to $<50.00 \mathrm{kUA} / \mathrm{L})$, Class 5 ( $\geq 50.00 \mathrm{kUA} / \mathrm{L}$ to $<$ $100.00 \mathrm{kUA} / \mathrm{L})$, and Class 6 ( $\geq 100.00 \mathrm{kUA} / \mathrm{L}) .{ }^{17}$

\subsection{Statistical analysis}

Data analyses were performed using the statistical software package SPSS 22.0 (IL, USA). Nonparametric quantitative data were described as medians (interquartile ranges), between-group comparisons of numerical data were performed using Mann-Whitney U tests or Kruskal-Wallis tests. Parametric quantitative data were depicted as means \pm standard deviations. To show the proportion of positive results, categorical data were reported as percentages. Chi-square $\left(\chi^{2}\right)$ tests or F-tests were used to demonstrate differences in proportions between groups. Correlation analyses among the groups were performed by calculating the Spearman correlation coefficient $\left(r_{s}\right)$. The correlation between components was calculated with optimal scale analysis. Differences were regarded as statistically significant if the $P$ value was lower than 0.05 .

\section{3.results}

\subsection{Totality}

In AIR-NCRCRD, 7.30\% of general population (295/4033) were sensitive to A.fumigatus in Guanzghou, slgE level was 1.10 (0.54-3.79) kUA/L. The positive rate of A.fumigatus slgE was significantly higher in adults than in children $(10.50 \%$ vs. $2.20 \%, P<0.001)$, but there were no differences between men and women. (Table 1). In 18 ABPA patients, there were 11 females, and the average age were 44.2 (range: 19 68) year old. In 54 patients with Af-sensitized asthma, there were 12 female, and the average age were 49.1 (range: 21 - 79) year old. There was no significant difference in age and gender between them.

\subsection{Fungal sensitization between ABPA patients and Af-sensitized asthma patients}

There was no significant difference of A.fumigatus slgE levels between ABPA and Af-sensitized asthma patients $(P>0.05)$. In this study, $94.44 \%$ of ABPA patients and $87.04 \%$ of Af-sensitized asthma patients were slgE-positive to at least one fungal allergen among the P.chrysogenum, C.herbarum, M.racemosus, C.albicans, A.alternata, and H.halodes. High positive rates to P.chrysogenum were found in $94.44 \%$ of ABPA patients and in $77.78 \%$ Af-sensitized asthma patients. The positivity rates of $C$.herbarum $(88.89 \%$ vs $62.96 \%, P<0.05)$ and A.alternata $(72.22 \%$ vs $44.44 \%, P<0.05)$ were higher in patients with ABPA than in Af-sensitized asthma patients (Figure 1). Although slgE levels of P.chrysogenum, C.herbarum, M.racemosus, C.albicans, A.alternata, and H.halodes slgE were higher in ABPA patients than in Afsensitized asthma patients, there were no significant differences between two groups (Figure 2a).

\subsection{A.fumigatus component sensitization between ABPA patients and Af-sensitized asthma patients}

The positive rates of Asp $f 1$ ( $88.89 \%$ vs $59.26 \%, P<0.05)$, Asp $f 2$ (66.67\% vs $33.33 \%, P<0.05)$, Asp $f 4$ $(61.11 \%$ vs $33.33 \%, P<0.05)$ and Asp f $6(66.67 \%$ vs $14.81 \%, P<0.001)$ in ABPA patients were significantly higher than those in Af-sensitized asthma patients. 
As shown in Figure 2b, slgE levels of Asp f 1 [7.93 (1.40, 30.18) kUA/L vs. $0.18(1.18,10.65) \mathrm{kUA} / \mathrm{L}, P<$ 0.05], Asp f $4[3.17(0.10,17.65)$ kUA/Lvs. $0.03(0.18,5.07)$ kUA/L, $P<0.05]$ and Asp f $6[1.22(0.07,5.70)$ kUA/L vs. $0.01(0.05,0.61) \mathrm{kUA} / \mathrm{L}, P<0.001]$ in ABPA patients were higher than those in Af-sensitized asthma patients. The co-sensitization of five allergen components was shown in Figure $\mathbf{3}$, and there were 7 ABPA patients and 10 Af-sensitized asthma patients co-sensitization to Asp f 1, Asp f 2, Asp f 3, Asp f 4 and Asp $f \quad 6$ at the same time. Interestingly, among them, there were 100\% ABPA patients co-sensitization to P.chrysogenum and C.albicans (Table.2).

\subsection{Correlation analysis between A.fumigatus components and various mycotic allergens in ABPA patients and Af-sensitized asthma patients}

Spearman correlation analysis show that tlgE $\left(r_{s}=0.586, P<0.05\right)$, $P$. chrysogenum $\left(r_{s}=0.686, P<0.05\right)$, C.herbarum $\left(r_{s}=0.688, P<0.05\right)$, M.racemosus $\left(r_{s}=0.358, P<0.05\right)$, C.albicans $\left(r_{s}=0.492, P<0.05\right)$, A.alternata $\left(r_{s}=0.692, P<0.05\right)$, and H.halodes $\left(r_{s}=0.585, P<0.05\right)$ had correlation with A.fumigatus. A.fumigatus component Asp $\mathrm{f} 1\left(r_{s}=0.473, P<0.05\right)$, Asp f $2\left(r_{s}=0.553, P<0.05\right)$, Asp f $3\left(r_{s}=0.558, P<\right.$ 0.05), Asp f $4\left(r_{s}=0.646, P<0.05\right)$ and Asp $f 6\left(r_{s}=0.730, P<0.05\right)$ was correlated with tlgE. In addition, Asp f $2\left(r_{s}=0.653, P<0.05\right)$, Asp $f 3\left(r_{s}=0.478, P<0.05\right)$, Asp $f 4\left(r_{s}=0.540, P<0.05\right)$ and Asp $f 6\left(r_{s}=\right.$ 0.483, $P<0.05)$ was correlated with $P$.chrysogenum, Asp $f 2\left(r_{s}=0.524, P<0.05\right)$ and Asp f $6\left(r_{s}=0.537\right.$,

$P<0.05)$ was correlated with M.racemosus, Asp f $2\left(r_{s}=0.568, P<0.05\right)$ and Asp f $3\left(r_{s}=0.514, P<0.05\right)$ was correlated with A.alternata, other mycotic allergens hadn`t significant correlation with A.fumigatus components. Interestingly, the correlation between A.fumigatus and it's component Asp f 2 was the strongest (ABPA: $r_{s}=0.786$; Af-sensitized asthma: $\left.r_{s}=0.663\right)$. Optimal scale analysis was show that ABPA was more relevant to Af-components (Cronbach`s alpha = 90.7\%) (Figure 4).

\section{4.discussion}

Fungus is one of the important causes of respiratory diseases. Europe`s investigations have shown that the incidence of fungus-induced respiratory tract allergies is as high as $20-30 \%$ in an atopic population, reaching $6 \%$ in the general population. ${ }^{18-20}$

In our study, we found that $7.30 \%$ general population were sensitization to A. fumigatus in local. Guangzhou city is influenced by the East Asian monsoon season and has a humid and warm subtropical climate, with a relative air humidity of $68 \%$ and annual precipitation of more than $1,700 \mathrm{~mm} .^{21} \mathrm{This}^{2}$ contribute greatly to the proliferation and growth of fungi, which prefer humid and warm environments. Therefore, a high concentration of mycotic spores in indoor and outdoor air is one of the most important causes of allergic respiratory tract diseases in Guangzhou. ${ }^{22,23}$ we also found that the positive rate of A.fumigatus was significantly higher in adult patients than in children. This suggested that sensitization to A. fumigatus allergens may be more common in adults and that adults with asthma should avoid contact with A. fumigatus allergens to prevent disease progression to ABPA ${ }^{24}$. 
Interestingly, $94.44 \%$ of ABPA patients and $87.04 \%$ of Af-sensitized asthma patients were co-sensitization to at least one other fungal allergen. This is concurrent with previous reports by Chang et al. and Ezeamuzie et al. showing that A.fumigatus, C.albicans, and P.chrysogenum were fungal allergens with the highest co-sensitization rates among all allergenic fungi. ${ }^{3,5} \mathrm{SIgE}$ sensitization to fungal species reflected well their phylogenetic relationship, since IgE reactivity correlated better in closely related molds than with phylogenetically distant molds. ${ }^{25,26}$ This phenomenon suggests that there may be a cross-react between allergies to A.fumigatus and other fungi.

In a study conducted in Sweden in 2010 , Soeria-Atmadja et al. analyzed the associations among mycotic allergies in 688 patients who were allergic to fungi. They reported that A.fumigatus had extremely strong correlations with P. chrysogenum and H.halodes $\left(r_{s}=0.85\right.$ and 0.87 , respectively) and strong correlations with C.herbarum, C.albicans, and A.alternata $\left(0.60 \leq r_{s}<0.80\right) .{ }^{26}$ However, in the present study, A.fumigatus was strongly correlated with C.herbarum $\left(r_{s}=0.688\right)$ in ABPA and A.alternata $\left(r_{s}=0.692\right)$ in Af-sensitized asthma patients but not correlated with C.albicans $(P>0.05)$.

The difference between fungal and nonfungal allergens is that fungal allergens are more complex. They contain proteases, glycosidases, and protein products, which can easily lead to cross-reactions.

Therefore, exposure to a single mycotic spore is equivalent to exposure to all fungal allergens. ${ }^{27}$ For example, P.chrysogenum and A.fumigatus both belong to family Trichocomaceae. ${ }^{25}$ Interaction between serum anti-P.chrysogenum antibodies in patients with ABPA can be greatly inhibited by A. fumigatus, probably owing to the high similarity between the primary allergenic components of P.chrysogenum, i.e., alkaline and vacuolar serine proteases, and their homologous allergenic components in A.fumigatus (Asp $\mathrm{f} 13$ and Asp f 18). ${ }^{11}$ Furthermore, the allergenic component in A.alternata, manganese-dependent superoxide dismutase (MnSOD), is the primary cause of the cross-reaction with Asp $f 6{ }^{28}$

In addition, ribosomal proteins are allergenic constituents of A.fumigatus components (Asp $f 8$ and Asp $f$ 23), A.alternata components (Alt a 5 and Alt a 12), and C.herbarum components (Cla h 5 and Cla h 12). ${ }^{29}$ Enolases are allergenic constituents of the A.fumigatus component Asp $f 22$, A. alternata component (Alt a 6), and C.herbarum component (Cla h 6). ${ }^{29}$ Therefore, cross-reactivity among fungal allergens should be considered when diagnosing fungal allergies to determine the appropriate treatment regimen.

In addition, the positive rates of Asp $f$ 1, Asp $f$ 2, Asp $f 4$ and Asp $f 6$ in ABPA patients were significantly higher than those in Af-sensitized asthma patients in our study. Patients with ABPA were characterized by higher levels of IgE Abs to Asp $f$ 1, Asp $f 4$ and Asp $f 6$ than those of Af-sensitized asthma patients. Some studies have shown that the combination of Asp $f 1$ and Asp $f 2$ can be considered a specific allergenic component in diagnosing A. fumigatus sensitization. ${ }^{12}$ However, some other reports have shown that the slgE levels for Asp f 2, Asp f 4, and Asp $f 6$ were highly specific markers for ABPA diagnosis. Their levels were significantly higher in the serum of patients with ABPA than in the serum of patients with Afsensitized asthma patients. ${ }^{12,29}$ Therefore, in the presence of an A.fumigatus allergy, in-depth analysis of 
A.fumigatus components could help to differentiate ABPA from Af-sensitized asthma patients. The insufficient sample size was the main defect of this paper, need to be supplemented by follow-up studies.

\section{Conclusion}

In summary, this study is the first study to demonstrate the complex relationship between A.fumigatus component and various mycotic allergens in ABPA and Af-sensitized asthma patients from Guangzhou, Southern China. Asp $f$ 1, Asp $f$ 2, Asp $f 4$ and Asp $f 6$ in ABPA patients were significantly higher than those in Af-sensitized asthma patients, and connection with various mycotic allergens. This findings can expected to help local doctors in the diagnosis of fungal allergies, particularly in distinguished diagnosis between ABPA and Af-sensitized asthma.

\section{Declarations}

\section{Ethics approval and consent to participate}

Approval was obtained from the ethic committee of The First Affiliated Hospital of Guangzhou Medical University (Reference number: GYFYY-2016-73). The use of human serum samples was in accordance with the legislation in China and the wishes of donors, their legal guardians or next of kin, where applicable, who had offered written informed consent to using the serum samples for future unspecified research purposes.

\section{Consent for publication}

Not applicable

\section{Availability of data and material:}

The data that support these findings are available on reasonable request from the corresponding author BQS. Data are not publicly available due to concerns regarding research participant privacy.

\section{Competing interests}

The authors declare that they have no competing interests.

\section{Funding}

This study was supported by the National Natural Science Foundation of China (81802076 and 81871736), National Key Technology R\&D Program (2018YFC1311900), Guangdong Science and Technology Foundation (2019B030316028), Guangzhou Municipal Health Foundation (20191A011073), and Guangzhou Science and Technology Foundation (201804020043).

\section{Author's contributions}


Conceived and designed the experiments: BQS. Performed the experiments: NLW, HMH, PYZ, YL. Analyzed the data: WTL, ZHW. Wrote the paper: HSH, WTL. All authors read and approved the final manuscript.

\section{Acknowledgements}

We thank Shiquan Wu and the doctors and nurses at the department of Allergy and Clinical Immunology of the First Affiliated Hospital of Guangzhou Medical University, China for their great support.

\section{References}

1.

Lin WR, Chen YH, Lee MF, et al. Does spore count matter in fungal allergy? The role of allergenic fungal species. Allergy Asthma Immunol Res. 2016;8:404-11.

2.

Twaroch TE, Curin M, Valenta R, et al. Mold allergens in respiratory allergy: From structure to therapy. Allergy Asthma Immunol Res. 2015;7:205-20.

3.

Ezeamuzie Cl, Al-Ali S, Khan M, et al. IgE-mediated sensitization to mould allergens among patients with allergic respiratory diseases in a desert environment. Int Arch Allergy Immunol. 2000;121:300-7. 4.

Nolard N. Fungal allergies. Mediators Inflamm. 2001 Nov;11(6):294-5.

5 .

Wu G, Meng X, Zheng P, et al. Elevated serum levels of periostin in patients with Allergic Bronchopulmonary Aspergillosis.Mycoses. 2019 Sep; 62 (9): 780-789.

6.

Luo W, Hu H, Tang W, et al. Allergen sensitization pattern of allergic adults and children in Southern China: a survey based on real life data. Allergy Asthma Clin Immunol. 2019 Jul;24:15:42.

7.

Zeng G, Hu H, Zheng P, et al. The practical benefit of Phadiatop test as the first-line in vitro allergenspecific immunoglobulin $\mathrm{E}$ (slgE) screening of aeroallergens among Chinese asthmatics: a validation study. Ann Transl Med. 2018 Apr;6(8):151.

8.

Crameri R, Zeller S, Glaser AG, Vilhelmsson M, Rhyner C. Cross-reactivity among fungal allergens: a clinically relevant. phenomenon? Mycoses. 2008;52:99-106.

9.

Canonica GW, Ansotegui IJ, Pawankar R, et al. A WAO-ARIA-GALEN consensus document on molecularbased allergy diagnostics. World Allergy Organ J. 2013;6:1.

10. 
$\mathrm{Hu} \mathrm{H}$, Huang $\mathrm{H}$, Zheng $\mathrm{P}$, et al. The sensitization characteristics of adult Chinese patients diagnosed with chronic respiratory diseases.Asian Pac J Allergy. 2019 Jun 4.

11.

Simon-Nobbe B, Denk U, Pöll V, et al. The spectrum of fungal allergy. International Archives of Allergy Immunology. 2008;145(1):58-86.

12.

Hu H, Luo W, Wu Z, et al. A pilot study on the allergen-specific IgE to molecular components on polysensitized mite allergic asthmatic patients in Guangzhou, China. Mol Immunol. 2019 Jan;105:38-45. 13.

Zhang CM, Deng YF, Lai H, et al. The analysis of multiple allergen slgE of patients with allergic disease. Guang dong Med J. 2015;36:1037-9. (In Chinese).

14.

Song GC, Wang XY, Wang Z, et al. Association between serum allergens and asthma in children. Chin J Contemp Pediatr. 2015;17:806-10.

15.

Agarwal R, Chakrabarti A, Shah A, et al. Allergic bronchopulmonary aspergillosis: review of literature and proposal of new diagnostic and classification criteria. Clin Exp Allergy. 2013;43:850-73.

16.

Global Initiative for Asthma [Internet]. Global Strategy for Asthma Management and Prevention. [cited 2015]. Available from: www.ginasthma.org.

17.

Zeng G, Luo W, Zheng P, et al. Component-resolved diagnostic study of Dermatophagoides Pteronyssinus major allergen molecules in a southern Chinese cohort. J Investig Allergol Clin Immunol. 2015;25:343-51. 18.

Heinzerling L, Frew AJ, Bindslev-Jensen C, et al. Standard skin prick testing and sensitization to inhalant allergens across Europe-a survey from the GALEN network. Allergy. 2005;60:1287-300. 19.

Niemi MH, Rytkönen-Nissinen M, Jänis J, et al. Structural aspects of dog allergies: the crystal structure of a dog dander allergen Can f 4. Mol Immunol. 2014;61:7-15.

20.

Kurup VP, Shen HD, Banerjee B. Respiratory fungal allergy. Microbes Infect. 2000;2:1101-10. 21.

Sun $\mathrm{BQ}$, Chen DH, Zheng PY, et al. Allergy-related evidences in relation to serum IgE: data from the China state key laboratory of respiratory disease, 2008-2013. Biomed Environ Sci. 2014;27:495-505.

22.

Chen $\mathrm{DH}$, Jiang M, Li J, et al. Risk factors of acarines sensitization for respiratory allergic disease of children in Guangzhou urban districts. Int J Respirdica Bioeng. 2010;30:1029-33. (In Chinese). 23.

Sun BQ, Lai KF, Li J, et al. The survey of the common inhaled allergen in the bronchial asthmatic patients in Guangzhou area of China. J Modern Clin Med Bioeng. 2004;10:217-9. (In Chinese). 
24.

Zhang $\mathrm{L}, \mathrm{Hu} \mathrm{H}$. Progress in diagnosis and treatment of allergic bronchial pulmonary aspergillosis. Chin $\mathrm{J}$ Tubere Respir Dis. 2011;34:688-90..(In Chinese).

25.

Teresa E, Twaroch M, Curin R, Valenta, et al. Mold Allergens in Respiratory Allergy: From Structure to Therapy. Allergy Asthma Immunol Res. 2015;7:205-20.

26.

Soeria-Atmadja D, Önell A, Borgå Å. IgE sensitization to fungi mirror fungal phylogenetic systematic. J Allergy Clin Immunol. 2010;125:1379-86.

27.

Brouwer J. Cross-reactivity between Aspergillus fumigatus and Penicillium. Int Arch Allergy Immuno. 1996;110:166-73.

28.

Gabriel MF, Postigo I, Gutierrez-Rodriguez A, et al. Characterisation of Alternaria alternata manganesedependent superoxide dismutase, a cross-reactive allergen homologue to Asp $\mathrm{f} 6$. Immunobiology. 2015;220:851-8.

29.

Kespohl S, Raulf M. Mould allergens: where do we stand with molecular allergy diagnostics?: Part 13 of the series Molecular Allergology. Allergol J Int. 2014;23:120-5.

\section{Tables}

Table 1. A.fumigatus sensitization in the study population

A. fumigatus positive rate, $\%(\mathrm{n} / \mathrm{N})$

\begin{tabular}{ll}
\hline Positive rates & $7.3(295 / 4033)$ \\
Sex & \\
Female & $7.9(184 / 2320)$ \\
Male & $6.5(111 / 1713)$ \\
Age & \\
$\leq$ 14 years & $2.2(34 / 1552)$ \\
$>14$ years & $10.5(261 / 2481)$ \\
sIgE class & \\
Class 1 & $34.9(103 / 295)$ \\
Class 2 & $38.0(112 / 295)$ \\
Class 3 & $15.6(46 / 295)$ \\
Class 4 & $9.8(29 / 295)$ \\
Class 5 & $1.0(3 / 295)$ \\
Class 6 & $0.7(2 / 295)$ \\
\hline
\end{tabular}

The A.fumigatus allergen sIgE positivity rate was significantly higher in adults than in children $(10.5 \%$ vs. $2.2 \%, P<0.001)$. 
Table.2 The sIgE level of A.fumigatus components and various mycotic allergens in ABPA patients which co-sensitization to Asp f 1 , Asp $\mathrm{f} 2$, Asp $\mathrm{f} 3$, Asp f 4 and Asp f 6

\begin{tabular}{|c|c|c|c|c|c|c|c|c|c|c|c|c|}
\hline 1 & 28.80 & 5.28 & 35.30 & 1.74 & 7.86 & 7.72 & 13.5 & 23.90 & 4.52 & 30.60 & 60.80 & 6.39 \\
\hline 2 & 9.28 & 2.46 & 15.90 & 0.80 & 8.23 & 5.07 & 2.28 & 6.03 & 6.89 & 28.20 & 5.58 & 13.80 \\
\hline 3 & 10.40 & 1.08 & 6.30 & 1.46 & 1.72 & 2.72 & 1.27 & 0.75 & 4.71 & 4.98 & 0.75 & 0.39 \\
\hline 4 & 100.00 & 1.49 & 12.40 & 4.86 & 5.38 & 2.56 & 1.61 & 1.58 & 8.53 & 0.30 & 27.70 & 5.48 \\
\hline 5 & 44.10 & 1.09 & 27.60 & 3.13 & 8.62 & 2.18 & 2.49 & 100.00 & 10.90 & 12.80 & 100.00 & 6.34 \\
\hline 6 & 5.93 & 1.95 & 40.60 & 0.21 & 1.00 & 2.15 & 0.50 & 42.40 & 16.80 & 11.10 & 80.10 & 4.12 \\
\hline 7 & 2.76 & 0.16 & 8.61 & 0.09 & 0.46 & 0.11 & 0.31 & 8.94 & 0.42 & 6.73 & 8.43 & 3.98 \\
\hline
\end{tabular}

\section{Figures}




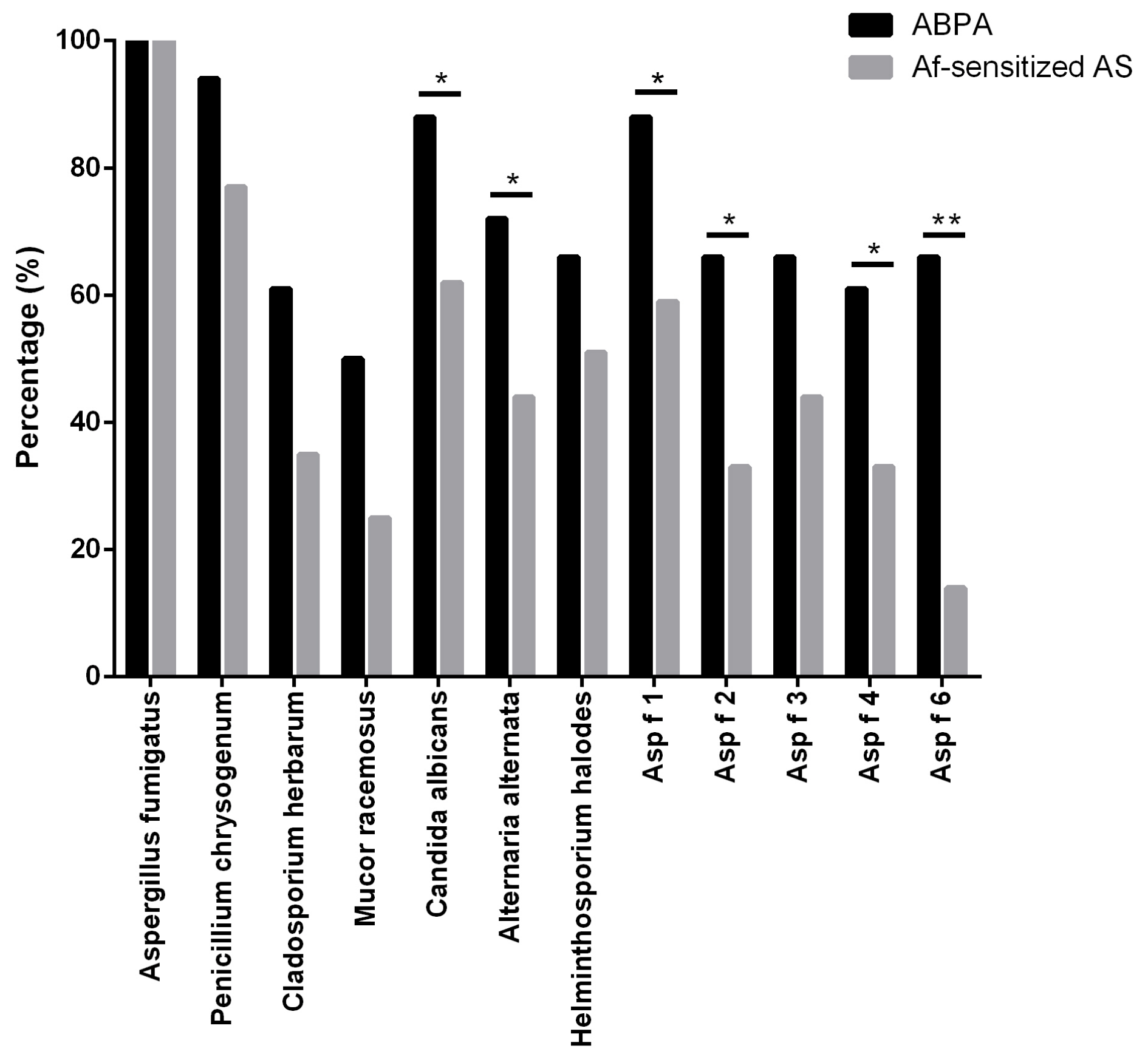

Figure 1

The positive rate of fungal allergens and A.fumigatus components between ABPA and A.fumigatus sensitized asthma patients ABPA: allergic bronchopulmonary aspergillosis; Af-sensitized AS: A.fumigatus sensitized asthma patients 


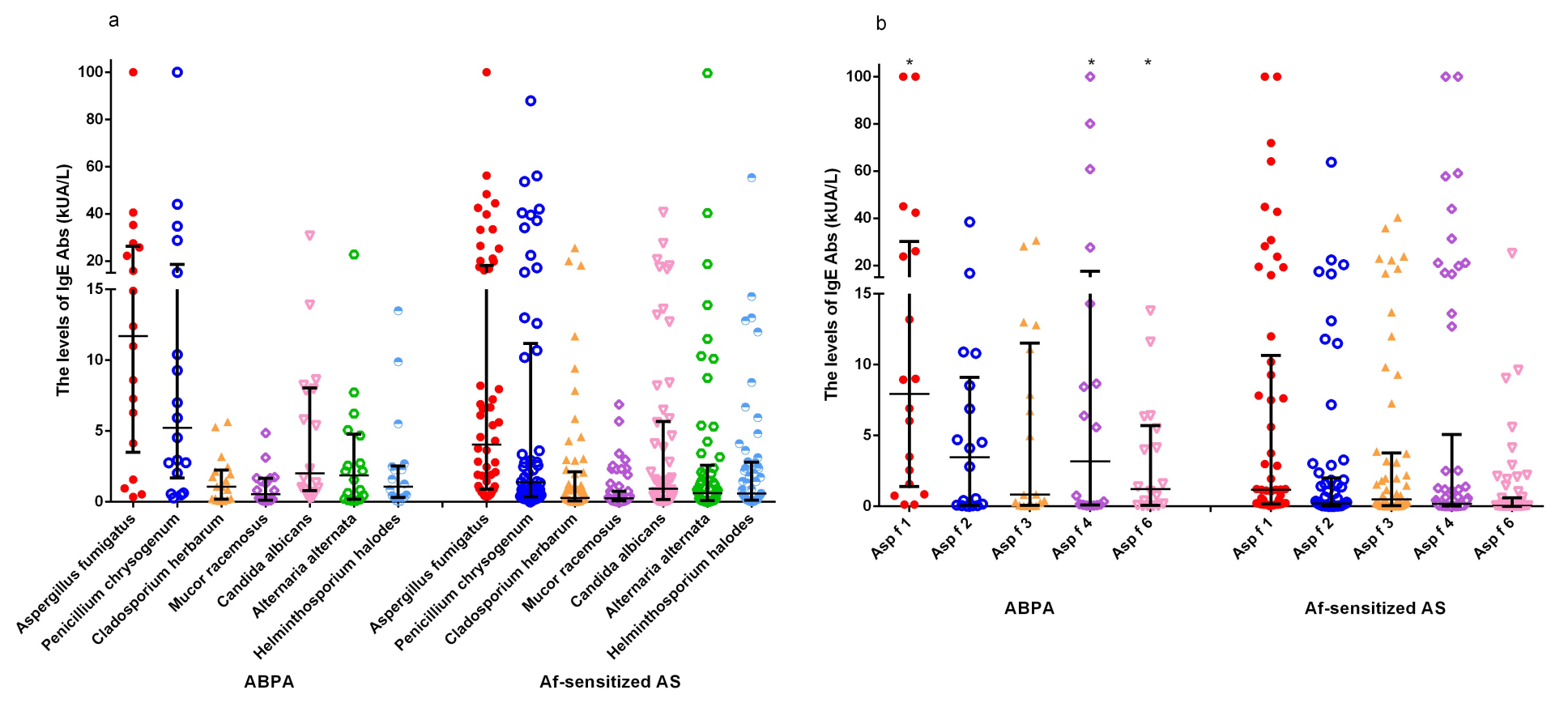

Figure 2

The slgE levels of fungal allergens and A.fumigatus components between ABPA and A.fumigatus sensitized asthma patients (a) fungal allergens; (b) A.fumigatus components ABPA: allergic bronchopulmonary aspergillosis; Af-sensitized AS: A.fumigatus sensitized asthma patients.
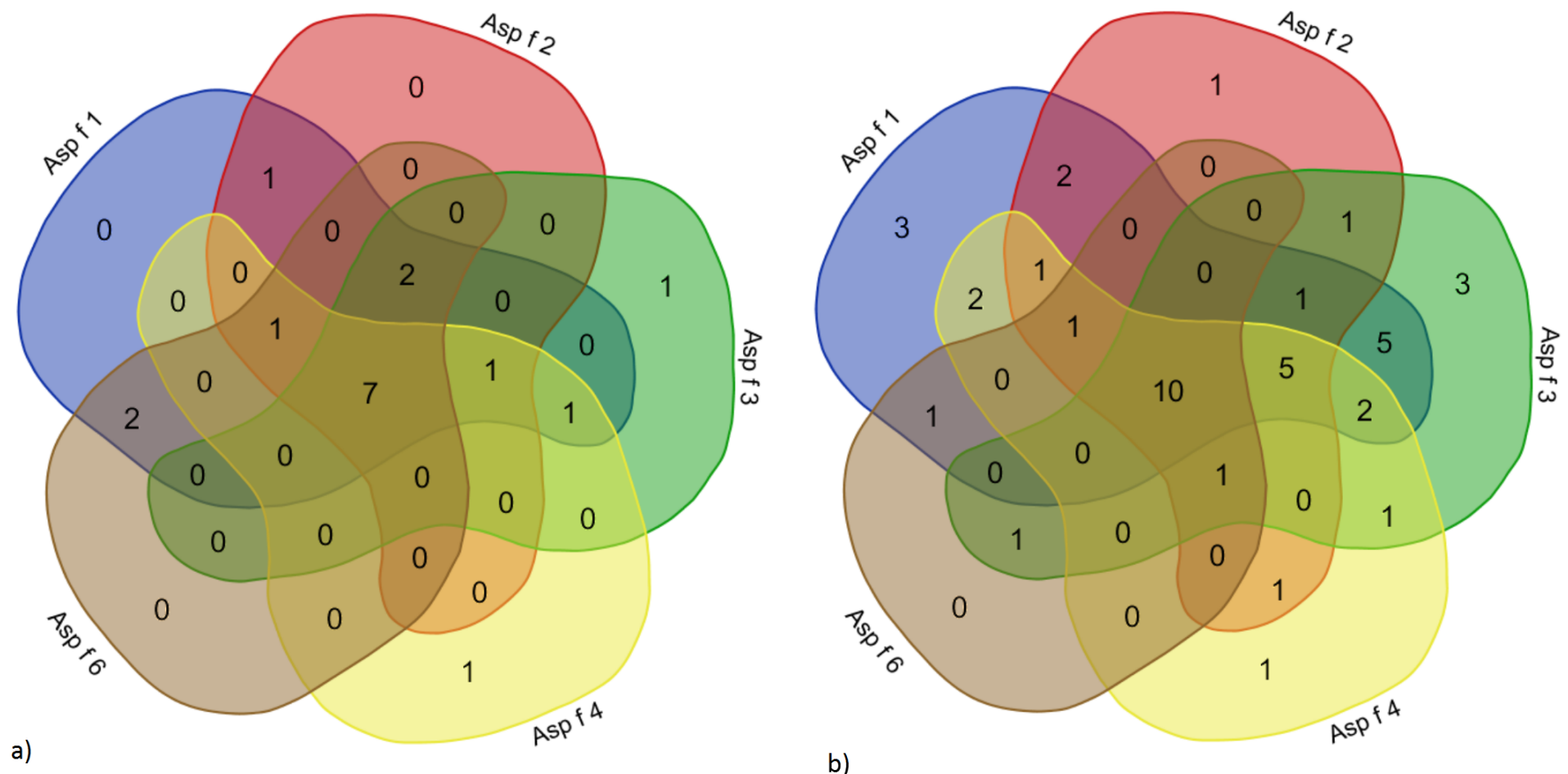

\section{Figure 3}

Co-sensitization of A.fumigatus-components. (a) Allergic bronchopulmonary aspergillosis; (b) A.fumigatus sensitized asthma patients 


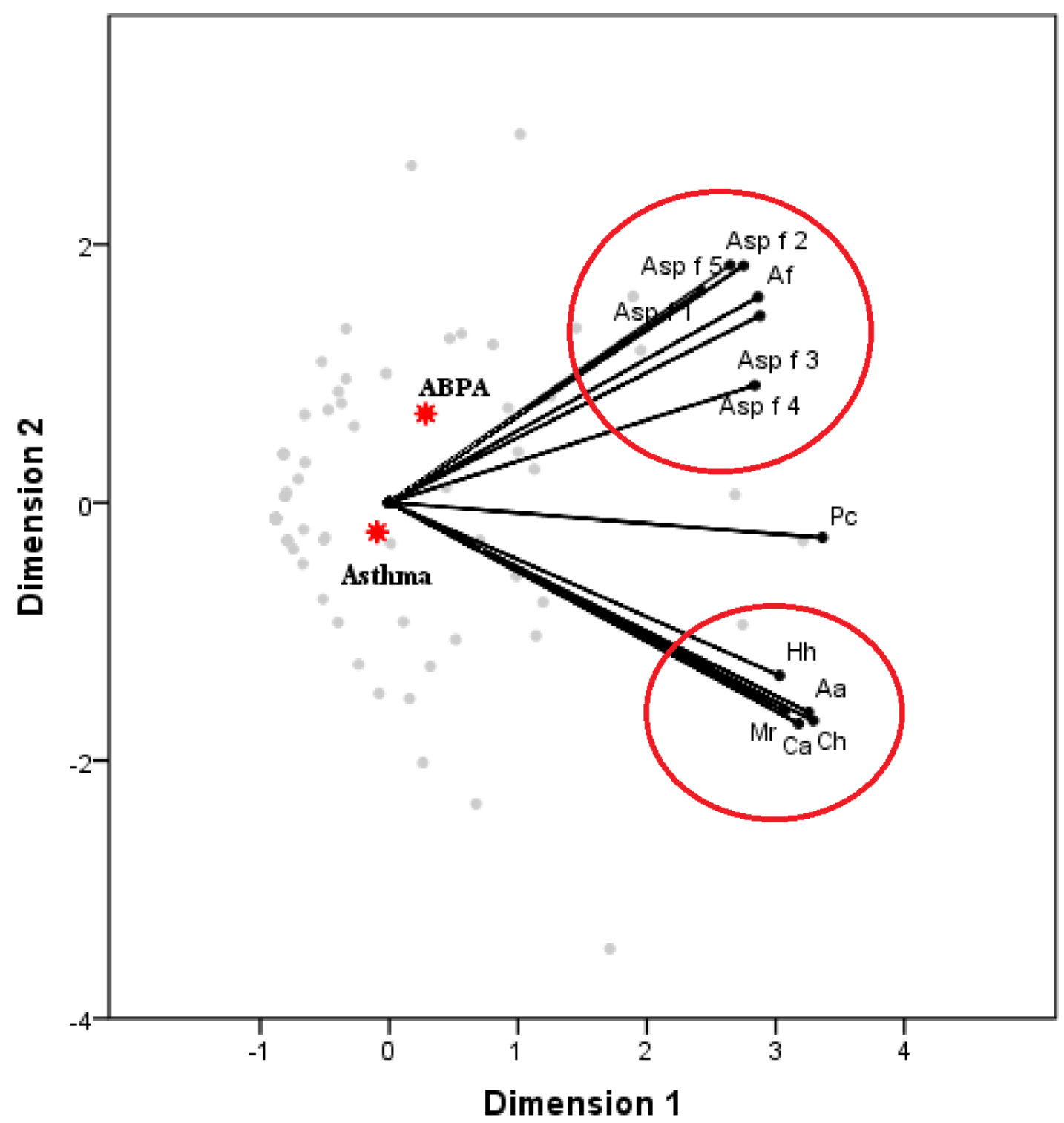

Figure 4

The optimal scale analysis of fungal allergens and A. fumigatus components The closer the distance between points, the closer the relationship is. Contrast to Af-sensitized asthma patients, ABPA was more relevant to Af-components. (Cronbach`s alpha $=90.7 \%$ ). Pc: P.chrysogenum; Ch: C.herbarum; Af: A.fumigatus; Mr: M.racemosus; Ca: C.albicans; Aa: A.alternata; Hh: H.halodes 